Published as: Auer, M., Schneeberger, A. \& Griffiths, M.D. (2012). Theoretical loss and gambling intensity: A simulation study. Gaming Law Review and Economics, 16, 269-273.

Theoretical loss and gambling intensity: a simulation study

Michael Auer, Andreas Schneeberger

a2mlab ltd

Office 404 Albany House

324 Regent Street

London

W1B 3HH

m.auer@a2mlab.com

\author{
Mark D. Griffiths, \\ Professor of Gambling Studies \\ International Gaming Research Unit \\ Psychology Division \\ Nottingham Trent University \\ Burton Street \\ Nottingham \\ NG1 4BU
}

mark.griffiths@ntu.ac.uk 


\begin{abstract}
Many recent studies of internet gambling-particularly those that have analysed behavioural tracking data-have used variables such as 'bet size' and 'number of games played' as proxy measures for 'gambling intensity.' In this paper, it is argued that the best and most stable measure for Gambling Intensity is the 'Theoretical Loss' (a product of total bet size and house advantage). In the long run, Theoretical Loss corresponds with the Gross Gaming Revenue generated by commercial gaming operators. For shorter periods of time, Theoretical Loss is the most stable measure of gambling intensity as it is not distorted by gamblers' occasional wins. Even for single bets, the Theoretical Loss reflects the amount a player is willing to risk. Using a simulation study, with up to 300,000 players playing as many as 13 different games, this paper demonstrates that the bet size and the number of games do not explain the theoretical loss entirely. In fact, there is a large proportion of variance which remains unexplained by measures of 'bet size' and 'number of games' played. Bet size and the number of games played do not equate to or explain theoretical loss, as neither of these two measures takes into account the house advantage.
\end{abstract}




\section{Theoretical loss and gambling intensity: a simulation study}

Many recent studies of internet gambling-particularly those that have analysed behavioural tracking data-have used variables such 'bet size' and 'number of games played' as proxy measures for 'gambling intensity. ${ }^{1-6}$ However, neither bet size nor the number of games played takes into account the house advantage of a game. Players are risking less when they play games with low house advantages. A low house advantage, therefoe, corresponds to a high payout. Furthermore, data presented from these studies have typically been presented by game type (e.g., data are only presented from online sports bettors or online poker players). However, using a concise simulation analysis of online gamblers playing a variety of games, this short paper argues that bet Size cannot be reliably used across games and/or game types as a measure of Gambling Intensity.

Griffiths and Auer ${ }^{7}$ outlined the many advantages and disadvantages of using behavioural tracking data in the gambling studies field. The main advantages of behavioural tracking data are that it (a) provides a totally objective record of an individual's gambling behaviour on a particular online gambling website; (b) provides a record of events and can be revisited after the event itself has finished; and (c) usually comprises very large sample sizes. These are the main reasons that such data will be used here.

In this paper, we describe what we believe is the best and most stable measure for 'gambling intensity.. This measure is the 'theoretical loss.' In the long run, outcomes in games of chance are always dependent on the house advantage: games with a great house advantage lead to higher losses for the gambler, while games with a lesser house advantage lead to lower losses. For instance, lottery games typically have relatively high house advantages (e.g., 50\%), whereas casino games 
typically have relatively low house advantages: roulette games with a single 'zero [0]' on their wheels, for example, have a house advantage of $2.7 \%$.

The 'loss/win' variable_often referred to as the Gross Gaming Revenue (GGR)— is the difference between 'total bet' and 'total win.' However, as a measure of a player's gambling intensity, it is not suitable, as it is typically distorted by the occasional winning occurrences by gamblers, particularly in the short-term. In the very long run, GGR is a more reliable indicator of gambling intensity, as (statistically) it corresponds to the theoretical loss. This means that theoretical loss is the most reliable and robust indicator of gambling intensity. The theoretical loss of any given game is represented by the product of the bet size and the house advantage. Over very long periods of time, the theoretical loss corresponds to the GGR with increasing accuracy. The following formula shows the calculation of the theoretical loss for a bet on a single game $[g]$. The house advantage depends on the specific game in question.

\section{Theoretical Loss $=$ Total Bet ${ }_{g} \times$ House Advantage $g$}

The more diverse the gambling behaviour, the more the bet size will typically deviate from the theoretical loss. For players playing only one game, a high bet size will always be accompanied a high theoretical loss. However, if the gambling behaviour is more diverse, players with high bet sizes will not necessarily have a high theoretical loss. Given the reliance on variables such as bet size and/or number of games played as proxy measures for gambling intensity, this paper examines the properties of theoretical loss using a mathematical simulation study of up to 300,000 gamblers, playing as many as 13 different games. The hypothesis was that the robustness of bet size and number of games played as measures of gambling intensity would decline as gaming becomes more hybrid. 


\section{Method}

The authors programmed a procedure in the statistical package ' $R$ ' that simulates different numbers of players, playing as many as 13 different forms of gambling games with varying house advantages. A simulation study is most appropriate to show that the bet size and the number of games does not completely explain the theoretical loss. Since an empirical study with a sample of gamblers always carries the problem of validity. For this reason, the mathematical approach of a simulation study was chosen. (However, the authors also plan to publish a similar study with real-world online behavioural data.)

Game Types: As argued above, casino operators offer a wide variety of games with different house advantages. The house advantage is a key structural factor that influences game attractiveness to players and helps determine the casinos' revenue stream. High house advantages tend to be less attractive to the gambler but yield high profits. Based on our experience in the field of gambling, as well as our knowledge of the different gaming platforms, we chose 13 games to be part in the simulation study. Table 1 displays these 13 games and their house advantages. These house advantages are mean average values, because different operators sometimes modify games slightly. For example, slot machines and video poker, in particular, can vary greatly across operators. Therefore, we provided two house advantages each for slot machines and video poker.

Table 1: Game type and house advantage used in the simulation study

\begin{tabular}{|l|r|}
\hline Game Type & $\begin{array}{l}\text { House } \\
\text { Advantage }\end{array}$ \\
\hline Keno & $25.0 \%$ \\
\hline Big 6 Wheel & $10.0 \%$ \\
\hline Roulette (double zero) & $5.6 \%$ \\
\hline $\begin{array}{l}\text { Video poker (high } \\
\text { house advantage) }\end{array}$ & $4.8 \%$ \\
\hline Sports Betting & $4.5 \%$ \\
\hline
\end{tabular}




\begin{tabular}{|l|r|}
\hline $\begin{array}{l}\text { Slot (high house } \\
\text { advantage) }\end{array}$ & $3.3 \%$ \\
\hline Roulette (single zero) & $2.7 \%$ \\
\hline $\begin{array}{l}\text { Slot (low house } \\
\text { advantage) }\end{array}$ & $1.8 \%$ \\
\hline Baccarat (banker) & $1.7 \%$ \\
\hline Baccarat (player) & $1.6 \%$ \\
\hline Blackjack & $0.8 \%$ \\
\hline Craps (double odds) & $0.6 \%$ \\
\hline $\begin{array}{l}\text { Video poker (low } \\
\text { house advantage) }\end{array}$ & $0.5 \%$ \\
\hline
\end{tabular}

Viewing the different house advantages also reflects the problem of using bet size or number of games as a measure of gambling intensity. Betting 10 Euros on keno actually corresponds to betting almost 100 Euros on roulette with a single zero. However, to date, all published studies using behavioural tracking data have only used bet size as the measure of gambling intensity. Using the number of games as a measure of gaming intensity would be an even-worse measure, as it also neglects the amount bet.

Simulation Parameters: As mentioned above, the first advantage of a simulation study is that gamblers could play up to 13 different games with varying house advantages. A second advantage is that the simulation can be conducted with different numbers of players. We simulated up to 300,000 players, playing the 13 games. For each of the players, the simulation calculates a specific game-preference pattern. On real world platforms, it is known that gamblers also play a variety of different games ${ }^{8}$. Once the game preferences have been determined, up to 5,000 games per player were simulated. Each simulated player has an individual number of games simulated. The 'drawing' of games happens according to the game preference pattern. Finally, the bet size per game being played has to be determined. Each player's range of bet size follows in a specific interval. The minimum bet size for each player is a natural number drawn from the interval [1:100]. To compute the maximum bet size, a natural number between 0 and 100 is drawn and added to the minimum bet size. 
Data analysis: The data analysis was performed with the statistical package 'R.' $R$ is a language and environment for statistical computing and graphics. It is a GNU project that is similar to the $S$ language and environment that was developed at Bell Laboratories (formerly AT\&T, now Lucent Technologies).

\section{Results}

The explanatory quality of bet size and number of games with respect to the theoretical loss can most easily be determined by performing a correlation analysis. Table 2 shows the amount of variance of the theoretical loss explained by bet size and number of games played. Table 2 represents the results for different numbers of players, wagering on up to 5,000 single games and playing up to 13 different game types. The amount bet also varies from 1 to 200 monetary units. It may clearly be seen that the $R^{2}$ converges to a specific value for both parameters. The simulation showed that bet size explains $56 \%$ of the variance of the theoretical loss, while the number of games played explains $32 \%$ of the variance of theoretical loss. This means that when using bet size alone, $44 \%$ of the gambling behaviour remains unexplained. When using the number of games played alone, $68 \%$ of the variance is left unexplained. The error when using bet size or the number of games played is considerable; it is clearly not justified to use only one of these two indicators as a measure of gaming intensity. All conclusions that have been drawn from previous studies should therefore be treated with considerable caution, as a large amount of the behaviour has not been accounted for.

\section{Table 2: Explained proportion of Theoretical Loss by bet size and number of games played for different numbers of players}




\begin{tabular}{|r|r|r|}
\hline \multicolumn{1}{|r|}{$n$} & Bet size & $\begin{array}{l}\text { Number of } \\
\text { games played }\end{array}$ \\
\hline 500 & $59 \%$ & $38 \%$ \\
\hline 1,000 & $61 \%$ & $32 \%$ \\
\hline 5,000 & $58 \%$ & $34 \%$ \\
\hline 10,000 & $56 \%$ & $32 \%$ \\
\hline 50,000 & $56 \%$ & $34 \%$ \\
\hline 100,000 & $56 \%$ & $32 \%$ \\
\hline 200,000 & $56 \%$ & $32 \%$ \\
\hline 300,000 & $56 \%$ & $32 \%$ \\
\hline
\end{tabular}

Table 2 clearly shows that the explained variance converges towards the two values $56 \%$ (bet size) and 32\% (number of games played). Another flexible parameter is the number of single games being played per individual. In Table 2, this number was drawn from the uniform distribution [1:5,000]. In Table 3, the number of players simulated was fixed at 300,000, but the number of games played was varied. The first two values of $R^{2}(52 \%$ and $29 \%)$ are the results from restricting the maximum number of single games played per individual to 50. The higher the number of single games, the more valid the result. For up to 1,000 single games, the two values of $R^{2}$ remain stable (see Table 3 ).

Table 3: Explained proportion of Theoretical Loss by bet size and number of games played for different numbers of games played

\begin{tabular}{|r|r|c|}
\hline & \multicolumn{2}{|c|}{$\mathrm{R}^{2}$} \\
\hline & & $\begin{array}{l}\text { Number } \\
\text { of games } \\
\text { played }\end{array}$ \\
\hline \# games & Betsize & $29 \%$ \\
\hline
\end{tabular}




\begin{tabular}{|l|r|r|}
{$[1 ; ; 50]$} & & \\
\hline$[1 ; 100]$ & $55 \%$ & $31 \%$ \\
\hline$[1 ; 1,000]$ & $56 \%$ & $32 \%$ \\
\hline$[1 ; 2,500]$ & $56 \%$ & $32 \%$ \\
\hline$[1 ; 5,000]$ & $56 \%$ & $32 \%$ \\
\hline
\end{tabular}

Another way of demonstrating the difference between Theoretical Loss, bet size, and number of games played is via a contingency table (see Table 4). The contingency table was produced from the simulation comprising 300,000 players playing a maximum of 5,000 single games each. Players were grouped into 10 groups according to Theoretical Loss and bet size. There was a correlation between the two measures, but was clearly far from perfect. Of the 30,000 most gaming intense players with respect to Theoretical Loss, 14,375 of them are also among the most gaming intense players with respect to bet size (therefore, 15,625 are not). The difference is even greater for moderate gamblers. For example, of the 30,000 gamblers in Group 6 of the Theoretical Loss group (see Table 4), only 5,430 are also in Group 6 of the bet size group. The error here amounts to 82\% ((30,000$5,430) / 30,000)$.

Table 4: Contingency table of Theoretical oss and bet size

\begin{tabular}{|c|c|c|c|c|c|c|c|c|c|c|c|c|}
\hline & & \multicolumn{10}{|c|}{ bet size } & \multirow[b]{2}{*}{ Total } \\
\hline & & 1 & 2 & 3 & 4 & 5 & 6 & 7 & 8 & 9 & 10 & \\
\hline \multirow{6}{*}{ 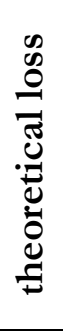 } & 1 & 23,687 & 5,371 & 742 & 143 & 39 & 15 & 1 & - & - & - & 29,998 \\
\hline & 2 & 5,583 & 13,384 & 7,295 & 2,403 & 838 & 303 & 132 & 46 & 16 & - & 30,000 \\
\hline & 3 & 668 & 7,195 & 8,941 & 6,987 & 3,622 & 1,528 & 639 & 262 & 121 & 37 & 30,000 \\
\hline & 4 & 53 & 3,085 & 6,178 & 6,999 & 6,267 & 4,026 & 1,981 & 903 & 364 & 144 & 30,000 \\
\hline & 5 & 7 & 787 & 4,299 & 5,275 & 5,896 & 5,721 & 4,167 & 2,382 & 1,070 & 396 & 30,000 \\
\hline & 6 & - & 144 & 1,885 & 4,419 & 4,777 & 5,430 & 5,440 & 4,445 & 2,555 & 905 & 30,000 \\
\hline
\end{tabular}




\begin{tabular}{|r|r|r|r|r|r|r|r|r|r|r|r|r|} 
& $\mathbf{7}$ & - & 31 & 533 & 2,744 & 4,466 & 4,665 & 5,230 & 5,433 & 4,565 & 2,333 & 30,000 \\
\cline { 2 - 13 } & $\mathbf{8}$ & - & 3 & 105 & 843 & 3,022 & 4,778 & 5,152 & 5,594 & 6,017 & 4,486 & 30,000 \\
\cline { 2 - 13 } & $\mathbf{9}$ & - & - & 20 & 170 & 943 & 3,022 & 5,467 & 6,348 & 6,706 & 7,324 & 30,000 \\
\cline { 2 - 12 } & $\mathbf{1 0}$ & - & - & 2 & 17 & 130 & 512 & 1,791 & 4,587 & 8,586 & 14,375 & 30,000 \\
\hline & Total & 29,998 & 30,000 & 30,000 & 30,000 & 30,000 & 30,000 & 30,000 & 30,000 & 30,000 & 30,000 & 299,998 \\
\hline
\end{tabular}

Table 5 shows that the concordance between Theoretical Loss and number of games is even less. Of the 30,000 most gaming intense players with respect to Theoretical Loss, only 8,649 are also among the most gaming intense players with respect to bet size (therefore, 21,351 are not). This amounts to an error of $71 \%$ $((30,000-8,649) / 30,000)$.

Table 5: Contingency table of Theoretical Loss and the number of games played

\begin{tabular}{|c|c|c|c|c|c|c|c|c|c|c|c|c|}
\hline & & \multicolumn{10}{|c|}{ bet size } & \multirow[b]{2}{*}{ Total } \\
\hline & & 1 & 2 & 3 & 4 & 5 & 6 & 7 & 8 & 9 & 10 & \\
\hline \multirow{11}{*}{ 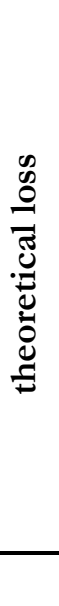 } & 1 & 20,203 & 5,220 & 1,949 & 974 & 574 & 351 & 247 & 174 & 142 & 100 & 29,934 \\
\hline & 2 & 6,783 & 9,199 & 5,169 & 2,954 & 1,980 & 1,287 & 913 & 735 & 523 & 457 & 30,000 \\
\hline & 3 & 2,108 & 6,859 & 6,275 & 4,425 & 3,121 & 2,291 & 1,785 & 1,253 & 1,062 & 821 & 30,000 \\
\hline & 4 & 646 & 4,333 & 5,509 & 4,986 & 3,998 & 3,085 & 2,452 & 2,086 & 1,590 & 1,315 & 30,000 \\
\hline & 5 & 175 & 2,511 & 4,220 & 4,772 & 4,472 & 3,851 & 3,163 & 2,699 & 2,233 & 1,904 & 30,000 \\
\hline & 6 & 34 & 1,218 & 2,988 & 4,143 & 4,285 & 4,314 & 3,976 & 3,421 & 3,003 & 2,618 & 30,000 \\
\hline & 7 & 4 & 536 & 2,173 & 3,336 & 3,844 & 4,318 & 4,245 & 4,126 & 3,853 & 3,565 & 30,000 \\
\hline & 8 & - & 142 & 1,226 & 2,503 & 3,421 & 4,193 & 4,574 & 4,684 & 4,711 & 4,546 & 30,000 \\
\hline & 9 & - & 35 & 425 & 1,567 & 2,930 & 3,827 & 4,527 & 5,068 & 5,659 & 5,962 & 30,000 \\
\hline & 10 & - & 2 & 68 & 385 & 1,268 & 2,552 & 4,115 & 5,678 & 7,283 & 8,649 & 30,000 \\
\hline & Total & 29,953 & 30,055 & 30,002 & 30,045 & 29,893 & 30,069 & 29,997 & 29,924 & 30,059 & 29,937 & 299,934 \\
\hline
\end{tabular}

\section{Discussion}

As far as the authors are aware, no paper published in the gambling studies literature has used Theoretical Loss as a measure of gambling intensity, and when gambling intensity has been examined, proxy measures, such as bet size and/or number of games played have been incorporated. The hypothesis that the 
robustness of bet size and number of games played as measures of gambling intensity would decline as gaming becomes more hybrid was confirmed.

This paper demonstrates that previous measures used are inadequate for explaining players' gambling intensity and that theoretical loss appears to be the most reliable and robust indicator of gaming intensity. The results presented using a concise simulation study clearly demonstrate that neither bet size nor (particularly) number of games played are robust measures of gambling intensity. This suggests that future studies should not consider such variables as proxy measures for gambling intensity, and that Theoretical Loss is a much more robust measure.

In order to make statements about overall gambling behavior, a complete view of gambling behavior has to be generated (including, for example, sports betting, casino games and poker). Results from previous studies outlined in the introduction were only derived singularly for sports betting, casino, or poker playing behavior. The analysis in the study presented here clearly demonstrates that researchers in the gambling studies field need to examine individual gambling behaviour across all game types and not just one type.

\section{References}

(1) A. Broda, D.A. LaPlante, S.E. Nelson, R.A., LaBrie, L.B. Bosworth \& H.J. Shaffer, Virtual harm reduction efforts for Internet gambling: effects of deposit limits on actual Internet sports gambling behaviour, 5 HARM REDUCTION JOURNAL 27 (2008).

(2) R.A. LaBrie, S. Kaplan, D.A. LaPlante, S.E. Nelson \& H.J. Shaffer, Inside the virtual casino: A prospective longitudinal study of Internet casino gambling, 18(4) EUROPEAN Journal of Public HeAlth 410-416 (2008).

(3) D.A. LaPlante, J.H., Kleschinsky, R.A., LaBrie, S.E. Nelson \& H.J. Shaffer, 
Sitting at the virtual poker table: A prospective epidemiological study of actual Internet poker gambling behavior, 25 COMPUTERS IN HUMAN BEHAVIOR 711-717 (2009).

(4) D.A. LaPlante, A. Schumann, R.A. LaBrie \& H.J. Shaffer, Population trends in Internet sports gambling, 24 COMPUTERS IN HUMAN BEHAVIOR 2399-2414 (2008).

(5) S.E. Nelson, D.A. LaPlante, A.J. Peller, A. Schumann, R.A. LaBrie \& H.J. Shaffer, Real limits in the virtual world: Self-limiting behavior of Internet gamblers, 24 JOURNAL OF GAMBLING STUdiES 463-477 (2008).

(6) S. Dragicevic, G. Tsogas \& A. Kudic, Analysis of casino online gambling data in relation to behavioural risk markers for bigh-risk gambling and player protection, 11 INTERNATIONAL GAMBLING STUDIES 377-391 (2011).

(7) M.D. Griffiths \& M. Auer, Online versus offline gambling: Methodological considerations in empirical gambling research, 7(3) CASINO AND GAMING INTERNATIONAL 45-48 (2011).

(8) H. Wardle, A. Moody, M.D. Griffiths, J. Orford \& R. Volberg, R. Defining the online gambler and patterns of behaviour integration: Evidence from the British Gambling Prevalence Survey 2010, 11 International Gambling Studies 339356 (2011). 